\title{
A CLASS OF BANACH ALGEBRAS WITH A UNIQUE NORM TOPOLOGY ${ }^{1}$
}

\author{
DAVID T. BROWN
}

The purpose of this paper is to produce a class of nonsemisimple Banach algebras with a unique norm topology. That is, a class of Banach algebras such that each member $B$ of the class has the property that any two Banach algebra norms on $B$ are equivalent (cf. [9, Chapter II]). (Throughout this paper, an algebra will denote any commutative algebra over the complex field $C$ which possesses an identity e.) To do this, we investigate an algebraic extension of a semisimple algebra $A$ which is similar to the Arens-Hoffman extension of a normed algebra (cf. [1]). We let $\alpha(x)$ be a monic polynomial in $A[x]$, the algebra of all polynomials in the indeterminate $x$ with coefficients in $A$, and denote by $(\alpha(x))$ the principal ideal in $A[x]$ generated by $\alpha(x)$. If $B=A[x] /(\alpha(x))$ is a Banach algebra with respect to some norm, then $A$ is a normed algebra with respect to the norm on $B$ restricted to $A$ and we ask whether or not $A$ is a closed subalgebra of $B$. If for any Banach algebra norm on $B, A$ is a closed subalgebra of $B$, then we show that $B$ has a unique norm topology.

The main result of this paper is that if $A$ is a regular (in the language of [9], completely regular), semisimple Banach algebra and if $\alpha(x)$ is any monic polynomial in $A[x]$, then $B=A[x] /(\alpha(x))$ has a unique norm topology, where $B$ is the Arens-Hoffman extension of $A$. (See below for a discussion of the Arens-Hoffman extension.) An example is given which shows that the condition of semisimplicity is essential. It is an open question whether or not the main result is true if $A$ is not a regular Banach algebra.

In the event that $A$ is a semisimple Banach algebra, $\alpha(x)$ is a monic polynomial in $A[x]$ and $B=A[x] /(\alpha(x))$ is semisimple, then by [9, Corollary 2.5.18], $B$ has a unique norm topology. Therefore our results have meaning only if $B$ is not semisimple. R. Arens and $\mathrm{K}$. Hoffman have shown that if the discriminant $d$ of $\alpha(x)$ is not a zero divisor in $A$, then $B$ is semisimple [1, Theorem 4.3]. (See [1, p. 207] for the definition of $d$.) The converse of this latter result is also valid

Received by the editors October 26, 1965.

1 The results in this paper form a portion of the author's doctoral dissertation written at Syracuse University under the direction of Professor John A. Lindberg, Jr., to whom the author wishes to express his appreciation. This research was partially supported by the U. S. Army Research Office in Durham and partially by a National Aeronautics and Space Administration Fellowship at Syracuse University. 
and is proved by $\mathrm{J}$. Lindberg [6, Corollary 9.3]. Therefore $B$ is not semisimple if and only if $d$ is either zero or a divisor of zero.

Unless otherwise stated, $A$ will denote a semisimple algebra, $\alpha(x)$ a monic polynomial in $A[x]$, and $B=A[x] /(\alpha(x))$. We will denote the coset $x+(\alpha(x))$ in $B$ by $x$ and the coset $a+(\alpha(x))$ by $a$, for each $a \in A$. Thus any element $b \in B$ can be uniquely expressed in the form $b=\sum_{i=0}^{n-1} a_{i} r^{i}$, where $n$ is the degree of $\alpha(x)$ and $a_{0}, a_{1}, \cdots, a_{n-1}$ are elements in $A$.

We begin by presenting a brief discussion of the Arens-Hoffman extension. If $A$ is a normed algebra (not necessarily semisimple), and $\alpha(x)$ is a monic polynomial in $A[x]$, Arens and Hoffman have shown in [1] that $B=A[x] /(\alpha(x))$ possesses a family of norms each of which makes $B$ into a normed algebra with the property that the natural embedding of $A$ into $B$ is an isometry as well as an isomorphism of $A$ onto a closed subalgebra of $B$. Furthermore, $B$ is a Banach algebra whenever $A$ is a Banach algebra. If $\alpha(x)=x^{n}+\sum_{i=0}^{n-1} \alpha_{i} x^{i}$, then the family of norms is given by

$$
\left\|\sum_{i=0}^{n-1} a_{i} i^{i}\right\|=\sum_{i=0}^{n-1}\left\|a_{i}\right\| t^{i}
$$

where $\|\cdot\|$ is the norm on $A$ and $t$ is any positive number such that $\sum_{i=0}^{n-1}\left\|\alpha_{i}\right\| t^{i} \leqq t^{n}$. Since there always exists a $t$ with the above property, we shall assume $t=1$. We will refer to $B$ as an Arens-Hoffman extension of $A$ and call the norm

$$
\left\|\sum_{i=0}^{n-1} a_{i} \mathfrak{x}^{i}\right\|=\sum_{i=0}^{n-1}\left\|a_{i}\right\| t^{i},
$$

for any appropriate $t$, an Arens-Hoffman norm.

We denote by $\Phi_{A}$ the carrier space of the normed algebra $A$ and by $\hat{A}$ the Gelfand representation of $A$ (cf. [9, Chapter III]). If $h \in \Phi_{A}$, we view $x$ as an indeterminate over $C$ as well as an indeterminate over $A$ and let $\alpha_{h}(x)$ denote the polynomial $\sum_{i=0}^{n-1} \hat{\alpha}_{i}(h) x^{i}$ in $C[x]$. Arens and Hoffman have shown that if $A$ is a normed algebra and if $\alpha(x)$ is a monic polynomial in $A[x]$, then $\Phi_{B}(B=A[x] /(\alpha(x)))$ is identifiable with the set $\left\{(h, \lambda) \in \Phi_{A} \times C: \alpha_{h}(\lambda)=0\right\}$ together with the relative topology of $\Phi_{A} \times C$ [1, Theorem 4.2]. The Arens-Hoffman extension has been studied by G. A. Heuer in [4], J. A. Lindberg in [6], [7], and [8], and by Heuer and Lindberg in [5].

We now return to our assumption that $A$ is a semisimple algebra. Our first result shows that if $B$ is a Banach algebra, then there exists a norm on $A$ with respect to which $A$ is a Banach algebra. 
Lemma 1. Assume $B$ is a Banach algebra with norm $\|\cdot\|$. Then there is a norm $\|\cdot\|^{\prime}$ on $A$ with respect to which $A$ is a Banach algebra. Also, $\|\cdot\|{ }^{\prime}$ is majorized by the norm in $B$ restricted to $A$.

Proof. Let $R$ be the radical of $B$. Since $\hat{B}$ is isomorphic to $B / R$, $\hat{B}$ is a Banach algebra with respect to the quotient norm $\|\hat{b}\|_{Q}$ $=\inf _{r \in R}\|b+r\|$. We will show that $\hat{A}$ is a $\|\cdot\|_{Q}$-closed subalgebra of $\hat{B}$. Assume $\left\{\hat{a}_{m}\right\}$ is a Cauchy sequence in $\hat{A}$. Then there is an element $b \in B$ such that $\left\|\hat{a}_{m}-\hat{b}\right\|_{Q} \rightarrow 0$ as $m \rightarrow \infty$. Since $\|\cdot\|_{\ell}$ majorizes the sup norm on $B,\left\|a_{m}-b\right\|_{\infty} \rightarrow 0$ as $m \rightarrow \infty$ and thus $\hat{b}$ is constant on each fiber of $\Phi_{B}$. For each $h \in \Phi_{A}$, denote the roots of $\alpha_{h}(x)=0$ by $\lambda_{1}(h)$, $\lambda_{2}(h), \cdots, \lambda_{n}(h)$, where each distinct root is repeated according to its multiplicity and $n$ is the degree of $\alpha(x)$. Also, let $s_{1}(h), s_{2}(h), \cdots$, $s_{n}(h)$ be the elementary symmetric functions of $\lambda_{1}(h), \lambda_{2}(h), \cdots, \lambda_{n}(h)$. Since

$$
\alpha_{h}(x)=\prod_{i=1}^{n}\left(x-\lambda_{i}(h)\right)=x^{n}+\sum_{j=1}^{n}(-1)^{j} s_{j}(h) x^{n-j}
$$

for each $h \in \Phi_{A}$,

$$
\hat{\alpha}(x)=x^{n}+\sum_{i=0}^{n-1} \hat{\alpha}_{i} x^{i}=x^{n}+\sum_{j=1}^{n}(-1)^{j_{s j}} x^{n-j} .
$$

But this means each $s_{j}$ is an element of $\hat{A}$ since $\hat{\alpha}$ is a polynomial over $\hat{A}$.

Now define a function $f$ on $\Phi_{A}$ by $f(h)=\sum_{j=1}^{n} \hat{b}\left(h, \lambda_{j}(h)\right)$ for each $h \in \Phi_{A}$. Clearly, $f \in C\left(\Phi_{A}\right)$. We will show that $f \in \hat{A}$. We view $f$ as a function on $\Phi_{B}$ by writing $f\left(h, \lambda_{i}(h)\right)=f(h)$ for any $\left(h, \lambda_{i}(h)\right) \in \Phi_{B}$. Thus $f$ is a symmetric function in the $\lambda_{j}(h)$ 's and can be expressed as a polynomial in the $s_{j}$ 's with coefficients in $\hat{A}$. Since each $s_{j} \in \hat{A}$, $f \in \hat{A}$. (This result is a special case of $[8$, Lemma 1.2].)

We have already shown that $\hat{b}$ is constant on the fibers of $\Phi_{B}$ and thus $f(h)=n \hat{b}\left(h, \lambda_{j}(h)\right)$ for any $j=1,2, \cdots, n$. Therefore $\hat{b} \in \hat{A}$. We have thus shown that $\hat{A}$ is a closed subalgebra of $\hat{B}$.

Now define $\|a\|^{\prime}=\|a\|_{Q}$ for all $a \in A$. Since $A$ is isomorphic to $\hat{A}$, $\|\cdot\|$ ' defines a norm on $A$ with respect to which $A$ is a Banach algebra. Furthermore, $\|a\|^{\prime}=\inf _{r \in R}\|a+r\| \leqq\|a\|$ and therefore the proof is complete.

We note that by $[9$, Corollary 2.5 .18$],\|\cdot\|^{\prime}$ is equivalent to any other Banach algebra norm on $A$.

Theorem 2. If $A$ has the property that for any Banach algebra norm on $B, A$ is a closed subalgebra of $B$, then $B$ has a unique norm topology. 
Proof. Assume $\|\cdot\|$ is a Banach algebra norm on $B$. By Lemma 1, $A$ is a Banach algebra with norm $\|\cdot\|^{\prime}$ and for each $a \in A,\|a\|^{\prime} \leqq\|a\|$. Since $A$ is a closed subalgebra of $B$, by [9, Corollary 2.5.18], there exists a constant $K$ such that $\|a\|^{\prime} \leqq\|a\| \leqq K\|a\|^{\prime}$ for each $a \in A$. Let $\|\cdot\|_{1}$ denote the Arens-Hoffman norm on $B$ which extends $\|\cdot\|^{\prime}$ on $A$, i.e. $\left\|\sum_{i=0}^{n-1} a_{i} \mathfrak{x}^{i}\right\|_{1}=\sum_{i=0}^{n-1}\left\|a_{i}\right\|^{\prime}$. To show $B$ has a unique norm topology, we show that $\|\cdot\|$ is equivalent to $\|\cdot\|_{1}$. If $M=\max _{0 \leq i \leq n-1}\left\|\mathfrak{r}^{i}\right\|$, then for any $a_{0}, a_{1}, \cdots, a_{n-1}$ in $A$,

$$
\left\|\sum_{i=0}^{n-1} a_{i} x^{i}\right\| \leqq M \sum_{i=0}^{n-1}\left\|a_{i}\right\| \leqq M K \sum_{i=0}^{n-1}\left\|a_{i}\right\|^{\prime}=M K\left\|\sum_{i=0}^{n-1} a_{i} \sum^{i}\right\|_{1},
$$

and, by the inverse mapping theorem, the two norms are equivalent. Therefore any norm on $B$ is equivalent to the Arens-Hoffman norm and the proof is complete.

We now prove two conditions on $A$ which are sufficient for $A$ to be a closed subalgebra of $B$. In the following theorem, we view $A$ as a function algebra. This involves no loss of generality since $A$ is assumed to be a semisimple algebra. Thus if $B$ is a Banach algebra, by Lemma $1, A$ is also a Banach algebra and therefore $A$ is a semisimple Banach algebra.

Theorem 3. Let $X$ be a compact Hausdorff space and let $A$ be a regular subalgebra of $C(X)$ which contains the constant functions. If $B$ is a Banach algebra with norm $\|\cdot\|$, then $A$ is a closed subalgebra of $B$.

Proof. By Lemma 1 , there exists a norm $\|\cdot\|^{\prime}$ on $A$ with respect to which $A$ is a Banach algebra and $\|a\|^{\prime} \leqq\|a\|$ for each $a \in A$. Since the identity mapping is an isomorphism of $A$ into $B$, by [2, Theorem 3.7 and Corollary 3.9], there is a finite set $F=\left\{h_{1}, h_{2}, \cdots, h_{k}\right\}$ in $\Phi_{A}$ such that for any neighborhood $V$ in $\Phi_{A}$ of $F$, there is a constant $M_{V}$ such that $\|a\| \leqq M_{V}\|a\|^{\prime}$ for all $a \in K(V)$, where $K(V)=\{c \in A$ : $\hat{c}=0$ on $V\}$ is the kernel of $V$. For any such $V$, let $u_{V} \in A$ be such that $\hat{u}_{V}=1$ on $V$.

To show $A$ is a closed subalgebra of $B$, let $\left\{a_{m}\right\}$ be a Cauchy sequence in $A$ with respect to $\|\cdot\|$. Thus there exists an element $b \in B$ such that $\left\|a_{m}-b\right\| \rightarrow 0$ as $m \rightarrow \infty$. But $\left\{a_{m}\right\}$ is also a Cauchy sequence with respect to $\|\cdot\|^{\prime}$ and hence there exists an element $c \in A$ such that $\left\|a_{m}-c\right\|^{\prime} \rightarrow 0$ as $m \rightarrow \infty$. For any neighborhood $V$ in $\Phi_{A}$ of $F, a_{m}-a_{m} u_{V}$ $\in K(V)$ for each $m=1,2, \cdots$, and thus $\left\|a_{m}-a_{m} u_{V}\right\|$ $\leqq M_{V}\left\|a_{m}-a_{m} u_{V}\right\|^{\prime}$. Therefore $\left\|a_{m}-a_{m} u_{V}-\left(c-c u_{v}\right)\right\| \rightarrow 0$ as $m \rightarrow \infty$ and hence $c-c u_{V}=b-b u_{V}$. Let $b_{0}, b_{1}, \cdots, b_{n-1}$ be in $A$ such that $b=\sum_{i=0}^{n-1} b_{i} x^{i}$ where $n$ is the degree of $\alpha(x)$. Then $c-c u_{V}=b_{0}-b_{0} u_{V}$ 
and for $i=1,2, \cdots, n-1, b_{i}=b_{i} u_{V}$ which means $\hat{b}_{i}=0$ on $\left\{h \in \Phi_{A}\right.$ : $\left.a_{V}(h) \neq 1\right\}$.

To show $b_{i}=0$ for $i=1,2, \cdots, n-1$, we first show $\hat{b}_{i}=0$ on $\Phi_{A}-F$. If $h_{0} \in \Phi_{A}-F$, let $V$ be a closed neighborhood in $\Phi_{A}$ of $F$ such that $h_{0} \notin V$ and let $u_{V} \in A$ such that $\hat{u}_{V}=1$ on $V$ and $u_{V}\left(h_{0}\right)=0$. These choices are possible since $\Phi_{A}$ is compact Hausdorff and $A$ is regular. Therefore $\hat{b}_{i}\left(h_{0}\right)=\hat{b}_{i}\left(h_{0}\right) \hat{u}_{V}\left(h_{0}\right)=0$ for $i=1,2, \cdots, n-1$, and since $h_{0}$ was arbitrary, $\hat{b}_{i}=0$ on $\Phi_{A}-F$. We next show that each $\hat{b}_{i}=0$ on $F$. Since each $\hat{b}_{i}$ is continuous on $\Phi_{A}$, each point $h_{j} \in F$ such that $\hat{b}_{i}\left(h_{j}\right) \neq 0$ for some $i=1,2, \cdots, n-1$ is an isolated point of $\Phi_{A}$. By relabeling these points if necessary, we may and do assume that there exists an integer $k^{\prime} \leqq k$ such that for each $j=1,2, \cdots, k^{\prime}$, there is an $i$ such that $1 \leqq i \leqq n-1$ and $\hat{b}_{i}\left(h_{j}\right) \neq 0$. Thus for each $j=1,2, \cdots, k^{\prime}$, there exists an idempotent $u_{j}$ in $A$ such that $a_{j}\left(h_{j}\right)=1$ and $a_{j}=0$ on $\Phi_{A}-\left\{h_{j}\right\}$. For any such $j$, consider the ideal $\left(u_{j}\right)$ in $A$ generated by $u_{j}$. This ideal is one-dimensional and thus is closed with respect to both norms in $A$. Since $a_{m} u_{j} \in\left(u_{j}\right)$ for each $m, b u_{j} \in\left(u_{j}\right) \subset A$. But $b u_{j}=\sum_{i=0}^{n-1} b_{i} u_{j} x^{i}$ and thus for each $i=1,2, \cdots, n-1, b_{i} u_{j}=0$. Therefore each $\hat{b}_{i}\left(h_{j}\right)=0$ and this means, since $j$ was arbitrary, that each $\hat{b}_{i}$ is identically zero on $F$. Since $A$ is semisimple, each $b_{i}=0$ and therefore $b=b_{0} \in A$. Thus $A$ is a closed subalgebra of $B$ and the proof is complete.

The techniques used in the above proof to show that each $\hat{b}_{\imath}$ is zero on $F$ are used to prove the following theorem.

Theorem 4. Assume $B$ is a Banach algebra with norm $\|\cdot\|$. If the isolated points of $\Phi_{A}$ are hull-kernel dense in $\Phi_{A}$, then $A$ is a closed subalgebra of $B$.

Proof. Let $I=\left\{h_{\gamma}: \gamma \in \Gamma\right\}$ be the isolated points in $\Phi_{A}$. For each $\gamma \in \Gamma$, let $e_{\gamma}$ be the idempotent in $A$ such that $\hat{e}_{\gamma}\left(h_{\gamma}\right)=1$ and $\hat{e}_{\gamma}=0$ on $\Phi_{A}-\left\{h_{\gamma}\right\}$. Let $\left\{a_{m}\right\}$ be a Cauchy sequence in $A$ and assume for $b=\sum_{i=0}^{n-1} b_{i} x^{i} \in B$ that $\left\|a_{m}-b\right\| \rightarrow 0$ as $m \rightarrow \infty$. For each $\gamma$, the ideal $\left(e_{\gamma}\right)$ in $A$ generated by $e_{\gamma}$ is one dimensional and hence is closed in $A$ with respect to the norm in $B$ restricted to $A$. Since $a_{m} e_{\gamma} \in\left(e_{\gamma}\right)$ for all $m, b e_{\gamma} \in\left(e_{\gamma}\right) \subset A$ and thus $b_{i} e_{\gamma}=0$ for $i=1,2, \cdots, n-1$. But this means that $\hat{b}_{i}\left(h_{\gamma}\right)=0$ and, since $\gamma$ was arbitrary, $b_{i} \in K(I)$ for $i=1,2, \cdots, n-1$. Therefore $\hat{b}_{i}=0$ on $H K(I)$, the hull of the kernel of $I$, which by assumption is $\Phi_{A}$. Finally, since $A$ is semisimple, $b_{i}=0$ and hence $b=b_{0} \in A$. This completes the proof.

We conclude with an example showing that the semisimplicity of $A$ is essential in Theorem 3 .

ExAmple. Let $A_{1}$ be any commutative Banach algebra without 
identity having the property that there exists a dense subalgebra $A_{0}$ of $A_{1}$ such that $A_{1}=A_{0} \oplus R$, where the radical of $A_{1}$ is $R$, and $R$ is a principal ideal generated by an element $r$ such that $r^{2}=r a=0$ for any $a \in A_{0}$. (See [2] or [3] for specific examples.) Thus $A_{0}$ is a Banach algebra with respect to the quotient norm $\|a\|_{Q}=\inf _{\gamma \in C}\|a+\gamma r\|$. Adjoin an identity $e$ to $A_{1}$ and obtain $A=A_{1} \oplus\{\lambda e\}$. Let $B=A[x] /\left(x^{2}\right)$ $=A \oplus A \mathfrak{x}$ and define a norm on $B$ as follows.

$$
\|a+\gamma r+\lambda e+(b+\mu r+\nu e) \mathfrak{x}\|_{1}=\|a+\mu r||+|\gamma|+|\lambda|+\| b \|_{Q}+|\nu|
$$

where $a, b \in A_{0}$. It is straightforward to verify that $B$ is a Banach algebra with norm $\|\cdot\|_{1}$. If we restrict $\|\cdot\|_{1}$ to $A$, we obtain $\|a+\gamma r+\lambda e\|_{1}$ $=\|a\|+|\gamma|+|\lambda|$. Since $A_{0}$ is not a closed subalgebra of $A_{1}$, there exists a Cauchy sequence $\left\{a_{m}\right\}$ in $A_{0}$ such that for some $a+\mu r$ in $A_{1}$ with $\mu \neq 0,\left\|a_{m}-a-\mu r\right\| \rightarrow 0$ as $m \rightarrow \infty$. Thus $\left\{a_{m}-a\right\}$ is a Cauchy sequence in $A$ which converges to $\mu r$ w with respect to $\|\cdot\|_{1}$. Therefore $A$ is not a closed subalgebra of $B$ with respect to $\|\cdot\|_{1}$.

\section{BiBLIOGRAPHY}

1. R. Arens and K. Hoffman, Algebraic extensions of normed algebras, Proc. Amer. Math. Soc. 7 (1956), 203-210.

2. W. G. Bade and P. C. Curtis, Homomorphisms of commutative Banach algebras, Amer. J. Math. 82 (1960), 589-608.

3. C. J. Feldman, The Wedderburn principle theorem in Banach algebras, Proc. Amer. Math. Soc. 2 (1951), 771-777.

4. G. A. Heuer, Algebraic extensions of Banach algebras, Ph.D. Thesis, Univ. of Minnesota, Minneapolis, Minn., 1958.

5. G. A. Heuer and J. A. Lindberg, Algebraic extensions of continuous function algebras, Proc. Amer. Math. Soc. 14 (1963), 337-342.

6. J. A. Lindberg, Algebraic extensions of commutative Banach algebras, Pacific J. Math. 14 (1964), 559-584.

7. - Factorization of polynomials over Banach algebras, Trans. Amer. Math. Soc. 112 (1964), 359-368.

8. - On the theory of algebraic extensions of a normed algebra, Ph.D. Thesis, Univ. of Minnesota, Minneapolis, Minn., 1960. 1960.

9. C. E. Rickart, General theory of Banach algebras, Van Nostrand, New York,

Hiram College 